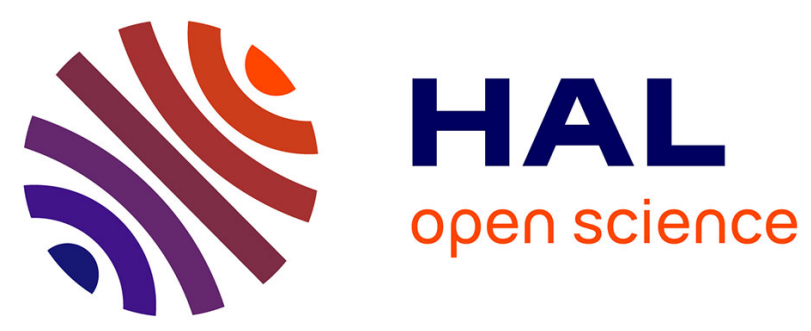

\title{
State observer design for Direct Contact Membrane Distillation Parabolic systems
}

Mohamed Ghattassi, Jean-Claude Vivalda, Taous Meriem Laleg-Kirati

\section{To cite this version:}

Mohamed Ghattassi, Jean-Claude Vivalda, Taous Meriem Laleg-Kirati. State observer design for Direct Contact Membrane Distillation Parabolic systems. ACC 2018 - American Control Conference, Jun 2018, Milwaukee, United States. 10.23919/ACC.2018.8431155 . hal-01876673

\section{HAL Id: hal-01876673 https://hal.inria.fr/hal-01876673}

Submitted on 12 Oct 2018

HAL is a multi-disciplinary open access archive for the deposit and dissemination of scientific research documents, whether they are published or not. The documents may come from teaching and research institutions in France or abroad, or from public or private research centers.
L'archive ouverte pluridisciplinaire HAL, est destinée au dépôt et à la diffusion de documents scientifiques de niveau recherche, publiés ou non, émanant des établissements d'enseignement et de recherche français ou étrangers, des laboratoires publics ou privés. 


\title{
State observer design for Direct Contact Membrane Distillation Parabolic systems
}

\author{
Mohamed Ghattassi, Jean-Claude Vivalda and Taous Meriem Laleg-Kirati, senior IEEE member
}

\begin{abstract}
This paper proposes a state observer for a coupled two dimensional partial differential equations (PDEs) system used to describe the heat transfer in a membrane distillation system for water desalination. The mathematical model based on reaction-diffusion system is introduced. Sufficient conditions for the exponential convergence of the estimation error is presented using Lyapunov method. A numerical example is also provided to illustrate the effectiveness of the proposed observer.
\end{abstract}

\section{INTRODUCTION}

Due to the increasing demand in potable water along with the limited natural freshwater resources, many countries rely on seawater desalination to meet their fresh water needs. However standard water desalination systems are usually energy inefficient and there is a pressing need to develop sustainable water desalination systems. Membrane distillation (MD) is an emerging technology for sustainable desalination process. It is a hybrid system with a membrane based thermal separation principle. It consists of two solutions: feed (hot sea water) and permeate (cold fresh water) separated by a micro-porous hydrophobic membrane which allows only water vapor to pass through the membrane pores from the feed side to the permeate side. When reaching the permeate surface, the water vapor condensates to produce fresh water [1], [2], [3]. The process in MD system is driven by the pressure gradient along the membrane sides, which is due to the temperature difference along the membrane boundaries. There are different MD configurations depending on a the nature of condensation procedure. This study focuses on the Direct-Contact Membrane Distillation (DCMD).

MD is a promising sustainable alternative to standard water desalination systems. This is mainly due to its potential to be directly powered by solar energy sources. However, its low production rate along with anomalies related to the presence of a membrane such as membrane fouling or wetting prevent from its commercialization [4]. Therefore, optimizing the MD systems by controlling and monitoring the system is needed. Recent work proposed accurate two dynamical mathematical models for the MD process [5],

M.Ghattassi, and T. M. Laleg-Kirati are with Computer Electrical and Mathematical Science and Engineering (CEMSE) Division, King Abdullah university of science and technology, KSA mohamed.ghattassilkaust.edu.sa; taousmeriem. laleg@kaust.edu.sa

J.C. Vivalda is with INRIA Villers-lès-Nancy, F-54600, France, University of Lorraine, IECL UMR CNRS 7502, 54506 Vandoeuvre-lès-Nancy, France. Jean-Claude.Vivalda@inria.fr
[6]. The first model is of distributed nature and is given by coupled Advection Diffusion equations representing the heat transfer mechanisms in both feed and permeate sides. The second model is a reduced order model describing the system's dynamics using a system of algebraic differential equations. Based on these two models, attempts of control and monitoring strategies have been proposed [7], [8]. However in most cases the temperature inside the MD module is required but not directly accessible for measurement [9], [10], [11]. In order to ease the control and monitoring process, in this paper we propose an observer based estimation of the MD temperature. For this purpose we are interested in the distributed model of the MD which consists of a two-dimensional reaction-diffusion system coupled at the boundary. In this model, we consider two control inputs which are the feed and the permeate inlet temperatures respectively, and two measured outputs for the DCMD process which are the feed and the permeate outlet temperatures respectively. Moreover the heat flux is also is assumed to be a measurement of the system. State observer is designed based on Lyapunov theory. Recently, a backstepping based observer has been proposed for reaction-diffusion processes evolving in multidimensional spatial domains. In this work, the observer design is developed for a class of reactiondiffusion system coupled on the boundary in two dimensional case.

The paper is organized as follows. Section 2, describes the MD model in DCMD system. Section 3 states the problem to be investigated and introduces the proposed observer structure with the underlying Lyapunov function. The estimation performance is illustrated through a numerical example in Section 4.

\section{Direct Contact Membrane Distillation SYSTEM}

\section{A. Mathematical Model}

The feed temperature $f$ is initially defined on the following domain $\left(0, \ell_{1}\right) \times\left(0, \ell_{2}\right)$ separated to the permeate by the membrane. The thickness of the membrane $\delta$ is very small which allow to assume that the spatial domain of the permeate temperature is given by $\left(\ell_{1}, 2 \ell_{1}\right) \times\left(0, \ell_{2}\right)$, see Fig.1. Finally, using the simple change of variables the feed and permeate temperatures are defined on some domain $\Omega=\left(0, \ell_{1}\right) \times\left(0, \ell_{2}\right)$. Let $T>0, Q_{T}=(0, T) \times \Omega$, $\Sigma_{\ell_{2}}=(0, T) \times\left(0, \ell_{2}\right)$ and $\Sigma_{\ell_{1}}=(0, T) \times\left(0, \ell_{1}\right)$. The mathematical model of the DCMD process is governed by 


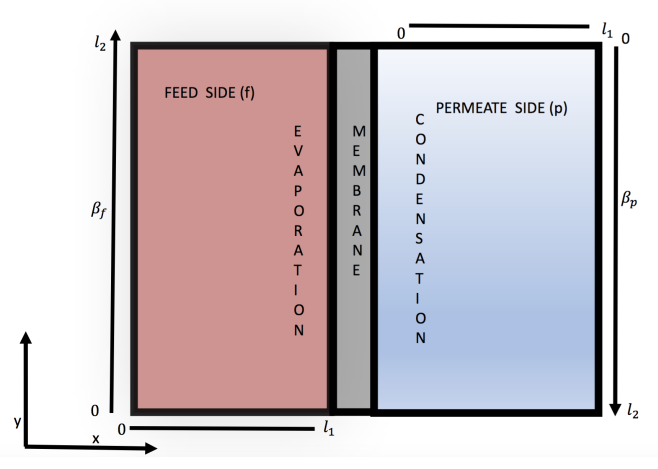

Fig. 1. Schematic diagram of DCMD process.

the following Parabolic system

$$
\begin{cases}\partial_{t} f-\alpha_{f} \partial_{x x} f+\beta_{f} \partial_{y} f=0 & \text { in } Q_{T}, \\ \partial_{t} p-\alpha_{p} \partial_{x x} p+\beta_{p} \partial_{y} p=0 & \text { in } Q_{T}, \\ \partial_{x} f\left(t, \ell_{1}, y\right)=-\frac{1}{\kappa_{f}}\left(\lambda J+\frac{\kappa_{m}}{\delta_{m}}(f-p)\right) & \text { in } \Sigma_{\ell_{2}}, \\ \partial_{x} f(t, 0, y)=0 & \text { in } \Sigma_{\ell_{2}}, \\ \partial_{x} p\left(t, \ell_{1}, y\right)=\frac{1}{\kappa_{p}}\left(\lambda J+\frac{\kappa_{m}}{\delta_{m}}(f-p)\right) & \text { in } \Sigma_{\ell_{2}}, \\ \partial_{x} p(t, 0, y)=0 & \text { in } \Sigma_{\ell_{2}}, \\ f(t, x, 0)=T_{f}(x) & \text { in } \Sigma_{\ell_{1}}, \\ p\left(t, x, \ell_{1}\right)=T_{p}(x) & \text { in } \Sigma_{\ell_{1}}, \\ f(t, x, y)=f_{0}(x, y) & \text { in } \Omega, \\ p(t, x, y)=p_{0}(x, y) & \text { in } \Omega,\end{cases}
$$

where $p_{0} \cdot f_{0} \in L^{2}(\Omega), T_{p}, T_{f} \in L^{2}\left(0, \ell_{1}\right), \alpha_{f}, \alpha_{p}>0$ be the thermal diffusivities, and $\beta_{f}>0, \beta_{p}<0$ be the velocities of flow in the feed and permeate side respectively. $k_{f}$ and $k_{p}$ are positive constants which depend on the thermal conductivity and thickness of the membrane. $\lambda$ is the latent heat of water. $J$ is the trans-membrane heat flux transferred between the feed and the permeate subsystems, it is based on Poiseuille-flow for the mass transfer coefficient

$$
J=C_{P} \Delta P=\frac{1}{8} \frac{r^{2} \epsilon}{\delta} \frac{M P_{m}}{\eta R T_{f p}}\left(P_{f}-P_{p}\right)
$$

where $P_{k}, k \in\{p, f\}$ the vapor pressure calculated using the Antoine equation

$$
P_{k}=\exp \left\{23.7836-\frac{3782.89}{k-42.85}\right\}
$$

with $k$ is the temperature in Kelvin $(\mathrm{K}), C_{P}$ is the Poiseuilleflow mass transfer coefficient, $T_{f p}=(f+p) / 2$ is the mean of the temperature, $P_{m}=\left(P_{f}+P_{p}\right) / 2$ is the mean pressure of the membrane and $\eta$ is the water vapor viscosity, $r$ is the pore size, $\epsilon$ is the porosity, $\tau$ is the tortuosity, $M$ is the water molecular weight and $R$ is the universal gas constant.

Remark 1: Initially, heat transfer in DCMD model is presented by the reaction diffusion parabolic system, under some reasonable physical assumptions given in [6] and [13, Chapter 10] we assume that the horizontal diffusivity is more affecting and has more significance than the vertical one. For this reason we neglected the vertical thermal diffusivity.

\section{B. Motivations}

The state-feedback controllers for the DCMD process require information on the state at each point in the domain. So, in the practice it is not possible to measure this state directly and we can measure only a small part of the state temperature for the feed $f\left(t, x, \ell_{2}\right)$ and permeate $p(t, x, 0)$ respectively which we are drawn to use observers for recovering the state from some measurements of the system. Moreover, the heat flux on the interface is known, therefore the flux $J$ is a measurement of system. This paper is devoted to the state observer of DCMD system where the goal is to propose an observer to estimate the temperature along of the membrane.

\section{OBSERVERS DESIGN}

Let introduce the following steady state system

$$
\begin{cases}-\alpha_{f} \partial_{x x} \bar{f}+\beta_{f} \partial_{y} \bar{f}=0 & \text { in } \Omega, \\ -\alpha_{p} \partial_{x x} \bar{p}-\beta_{p} \partial_{y} \bar{p}=0 & \text { in } \Omega, \\ \partial_{x} \bar{f}=-\frac{1}{\kappa_{f}}\left(\lambda J+\frac{\kappa_{m}}{\delta_{m}}(\bar{f}-\bar{p})\right) & \text { on }\left(0, \ell_{2}\right), \\ \partial_{x} \bar{p}=\frac{1}{\kappa_{p}}\left(\lambda J+\frac{\kappa_{m}}{\delta_{m}}(\bar{f}-\bar{p})\right) & \text { on }\left(0, \ell_{2}\right), \\ \partial_{x} \bar{f}=0 & \text { on }\left(0, \ell_{2}\right), \\ \partial_{x} \bar{p}=0 & \text { on }\left(0, \ell_{2}\right), \\ \bar{f}(x, 0)=T_{f}(x) & \text { on }\left(0, \ell_{1}\right), \\ \bar{p}\left(x, \ell_{2}\right)=T_{p}(x) & \text { on }\left(0, \ell_{1}\right),\end{cases}
$$

Let

$$
\overline{\bar{f}}=f-\bar{f} .
$$

Then,

$$
\begin{cases}\partial_{t} \overline{\bar{f}}-\alpha_{f} \partial_{x x} \overline{\bar{f}}+\beta_{f} \partial_{y} \overline{\bar{f}}=0 & \text { in } Q_{T}, \\ \partial_{t} \overline{\bar{p}}-\alpha_{p} \partial_{x x} \overline{\bar{p}}-\beta_{p} \partial_{y} \overline{\bar{p}}=0 & \text { in } Q_{T}, \\ \partial_{x} \overline{\bar{f}}\left(t, \ell_{1}, y\right)=-\gamma\left(\overline{\bar{f}}\left(t, \ell_{1}, y\right)-\overline{\bar{p}}\left(t, \ell_{1}, y\right)\right) & \text { on } \Sigma_{\ell_{2}}, \\ \partial_{x} \overline{\bar{p}}\left(t, \ell_{1}, y\right)=\gamma_{1}\left(\overline{\bar{f}}\left(t, \ell_{1}, y\right)-\overline{\bar{p}}\left(t, \ell_{1}, y\right)\right) & \text { on } \Sigma_{\ell_{2}}, \\ \partial_{x} \overline{\bar{f}}(t, 0, y)=0 & \text { on } \Sigma_{\ell_{2}}, \\ \partial_{x} \overline{\bar{p}}(t, 0, y)=0 & \text { on } \Sigma_{\ell_{2}}, \\ \overline{\bar{f}}(t, x, 0)=0 & \text { on } \Sigma_{\ell_{1}}, \\ \overline{\bar{p}}\left(t, x, \ell_{2}\right)=0 & \text { on } \Sigma_{\ell_{1}}, \\ \overline{\bar{f}}(0, y, x)=f_{0}(x, y)-\bar{f}(x, y) & \text { in } \Omega, \\ \overline{\bar{p}}(0, y, x)=p_{0}(x, y)-\bar{p}(x, y) & \text { in } \Omega,\end{cases}
$$

where $\gamma=\frac{\kappa_{m}}{\kappa_{f} \delta_{m}}$ and $\gamma_{1}=\frac{\kappa_{m}}{\kappa_{p} \delta_{m}}$. We now consider the following observer

$\begin{cases}\partial_{t} \hat{f}-\alpha_{f} \partial_{x x} \hat{f}+\beta_{f} \partial_{y} \hat{f}=L_{f}\left(\overline{\bar{f}}\left(t, x, \ell_{2}\right)-\hat{f}\left(t, x, \ell_{2}\right)\right) & \text { in } Q_{T}, \\ \partial_{t} \hat{p}-\alpha_{p} \partial_{x x} \hat{p}-\beta_{p} \partial_{y} \hat{p}=L_{p}(\overline{\bar{p}}(t, x, 0)-\hat{p}(t, x, 0)) & \text { in } Q_{T}, \\ \partial_{x} \hat{f}\left(t, \ell_{1}, y\right)=-\gamma\left(\hat{f}\left(t, \ell_{1}, y\right)-\hat{p}\left(t, \ell_{1}, y\right)\right) & \text { on } \Sigma_{\ell_{2}}, \\ \partial_{x} \hat{p}\left(t, \ell_{1}, y\right)=\gamma_{1}\left(\hat{f}\left(t, \ell_{1}, y\right)-\hat{p}\left(t, \ell_{1}, y\right)\right) & \text { on } \Sigma_{\ell_{2}}, \\ \partial_{x} \hat{f}(t, 0, y)=0 & \text { on } \Sigma_{\ell_{2}}, \\ \partial_{x} \hat{p}(t, 0, y)=0 & \text { on } \Sigma_{\ell_{2}}, \\ \hat{f}(t, x, 0)=0 & \text { on } \Sigma_{\ell_{1}}, \\ \hat{p}\left(t, x, \ell_{2}\right)=0 & \text { on } \Sigma_{\ell_{1}}, \\ \hat{f}(0, y, x)=\hat{f}_{0}(x, y) & \text { in } \Omega, \\ \hat{p}(0, y, x)=\hat{p}_{0}(x, y) & \text { in } \Omega,\end{cases}$


where $L_{f}$ and $L_{p}$ are the observers gains. Let us give the following error dynamic

$$
e^{f}=\hat{f}-\overline{\bar{f}}, \quad e^{p}=\hat{p}-\overline{\bar{p}} .
$$

Then, we have

$$
\begin{cases}\partial_{t} e^{f}-\alpha_{f} \partial_{x x} e^{f}+\beta_{f} \partial_{y} e^{f}=L_{f} e^{f}\left(t, x, \ell_{2}\right) & \text { in } Q_{T}, \\ \partial_{t} e^{p}-\alpha_{p} \partial_{x x} e^{p}-\beta_{p} \partial_{y} e^{p}=L_{p} e^{p}(t, x, 0) & \text { in } Q_{T}, \\ \partial_{x} e^{f}\left(t, \ell_{1}, y\right)=-\gamma\left(e^{f}\left(t, \ell_{1}, y\right)-e^{p}\left(t, \ell_{1}, y\right)\right) & \text { on } \Sigma_{\ell_{2}}, \\ \partial_{x} e^{p}\left(t, \ell_{1}, y\right)=\gamma_{1}\left(e^{f}\left(t, \ell_{1}, y\right)-e^{p}\left(t, \ell_{1}, y\right)\right) & \text { on } \Sigma_{\ell_{2}}, \\ \partial_{x} e^{f}(t, 0, y)=0 & \text { on } \Sigma_{\ell_{2}}, \\ \partial_{x} e^{f}(t, 0, y)=0 & \text { on } \Sigma_{\ell_{2}}, \\ e^{f}(t, x, 0)=0 & \text { on } \Sigma_{\ell_{1}}, \\ e^{p}\left(t, x, \ell_{2}\right)=0 & \text { on } \Sigma_{\ell_{1}}, \\ e^{f}(0, y, x)=e_{0}^{f}(x, y) & \text { in } \Omega, \\ e^{p}(0, y, x)=e_{0}^{p}(x, y) & \text { in } \Omega,\end{cases}
$$

where

$$
e_{0}^{f}=\hat{f}_{0}-\overline{\bar{p}}_{0}, \quad e_{0}^{p}=\hat{p}_{0}-\overline{\bar{p}}_{0} .
$$

We now introduce the main result of this paper which consists in a sufficient stability condition guaranteeing the exponential convergence of the estimation error towards zero.

Theorem 1: The estimation error (5) converges exponentially towards zero if there exist gain observers satisfying

$$
\left\{\begin{array}{c}
\left\|L_{f}\right\|_{\infty}^{2} \leqslant 2 \frac{\alpha_{f} \alpha_{p}^{2} \gamma_{1}^{2} \beta_{f}}{\ell_{2} c} \\
\text { and } \\
\left\|L_{p}\right\|_{\infty}^{2} \leqslant 2 \frac{\alpha_{p} \alpha_{f}^{2} \gamma^{2} \beta_{p}}{\ell_{2} c},
\end{array}\right.
$$

where $c=\left(\left(\ell_{1}+\ell_{2}\right)^{2} / \pi^{2}\right)$.

Proof: Let introduce the following Lyapunov function

$$
\begin{aligned}
V(t)= & \alpha_{p} \gamma_{1} \int_{0}^{\ell_{1}} \int_{0}^{\ell_{2}} e^{f 2}(t, x, y) d y d x \\
& +\alpha_{f} \gamma \int_{0}^{\ell_{1}} \int_{0}^{\ell_{2}} e^{p 2}(t, x, y) d y d x,
\end{aligned}
$$

where

$$
\begin{aligned}
V^{\prime}(t)= & 2 \alpha_{p} \gamma_{1} \int_{0}^{\ell_{1}} \int_{0}^{\ell_{2}} e_{f}(t, x, y) \partial_{t} e^{f}(t, x, y) d y d x \\
& +2 \alpha_{f} \gamma \int_{0}^{\ell_{1}} \int_{0}^{\ell_{2}} e^{p}(t, x, y) \partial_{t} e^{p}(t, x, y) d y d x .
\end{aligned}
$$

Then,

$$
\begin{aligned}
V^{\prime}(t) & =2 \alpha_{f} \alpha_{p} \gamma_{1} \int_{0}^{\ell_{1}} \int_{0}^{\ell_{2}} e^{f}(t, x, y) \partial_{x x} e^{f}(t, x, y) d y d x \\
& -2 \beta_{f} \alpha_{p} \gamma_{1} \int_{0}^{\ell_{1}} \int_{0}^{\ell_{2}} e_{f}(t, x, y) \partial_{y} e^{f}(t, x, y) d y d x \\
& +2 \alpha_{p} \gamma_{1} \int_{0}^{\ell_{1}} \int_{0}^{\ell_{2}} e_{f}(t, x, y) L_{f} e^{f}\left(t, x, \ell_{2}\right) d y d x \\
& +2 \alpha_{p} \alpha_{f} \gamma \int_{0}^{\ell_{1}} \int_{0}^{\ell_{2}} e^{p}(t, x, y) \partial_{x x} e^{p}(t, x, y) d y d x \\
& +2 \beta_{p} \alpha_{f} \gamma \int_{0}^{\ell_{1}} \int_{0}^{\ell_{2}} e^{p}(t, x, y) \partial_{y} e^{p}(t, x, y) d y d x \\
& +2 \alpha_{f} \gamma \int_{0}^{\ell_{1}} \int_{0}^{\ell_{2}} e^{p}(t, x, y) L_{p} e^{p}(t, x, 0) d y d x
\end{aligned}
$$

By integration by part, we have

$$
\begin{aligned}
\int_{0}^{\ell_{1}} & \int_{0}^{\ell_{2}} e^{f}(t, x, y) \partial_{x x} e^{f}(t, x, y) d y d x= \\
& \int_{0}^{\ell_{2}} e^{f}\left(t, \ell_{1}, y\right) \partial_{x} e^{f}\left(t, \ell_{1}, y\right) d y \\
& -\int_{0}^{\ell_{1}} \int_{0}^{\ell_{2}}\left(\partial_{x} e^{f}(t, x, y)\right)^{2} d y d x d y
\end{aligned}
$$

From boundary conditions we deduce

$$
\begin{aligned}
\int_{0}^{\ell_{1}} \int_{0}^{\ell_{2}} e^{f}(t, x, y) \partial_{x x} e^{f}(t, x, y) d y d x= \\
\quad-\gamma \int_{0}^{\ell_{2}} e^{f}\left(t, \ell_{1}, y\right)\left(e^{f}\left(t, \ell_{1}, y\right)-e^{p}\left(t, \ell_{1}, y\right)\right) d y \\
\quad-\int_{0}^{\ell_{1}} \int_{0}^{\ell_{2}}\left(\partial_{x} e^{f}(t, x, y)\right)^{2} d y d x .
\end{aligned}
$$

Similarly, we have

$$
\begin{gathered}
\int_{0}^{\ell_{1}} \int_{0}^{\ell_{2}} e^{p}(t, x, y) \partial_{x x} e^{p}(t, x, y) d y d x= \\
\gamma_{1} \int_{0}^{\ell_{2}} e^{p}\left(t, \ell_{1}, y\right)\left(e^{f}\left(t, \ell_{1}, y\right)-e^{p}\left(t, \ell_{1}, y\right)\right) d y \\
-\int_{0}^{\ell_{1}} \int_{0}^{\ell_{2}}\left(\partial_{x} e^{p}(t, x, y)\right)^{2} d y d x \\
-2 \beta_{f} \alpha_{p} \gamma_{1} \int_{0}^{\ell_{1}} \int_{0}^{\ell_{2}} e_{f}(t, x, y) \partial_{y} e^{f}(t, x, y) d y d x= \\
-\beta_{f} \alpha_{p} \gamma_{1} \int_{0}^{\ell_{1}}\left(e^{f}\right)^{2}\left(t, x, \ell_{2}\right) d x \\
2 \beta_{p} \alpha_{f} \gamma \int_{0}^{\ell_{1}} \int_{0}^{\ell_{2}} e_{p}(t, x, y) \partial_{y} e^{p}(t, x, y) d y d x= \\
-\beta_{p} \alpha_{f} \gamma \int_{0}^{\ell_{1}}\left(e^{p}\right)^{2}(t, x, 0) d x
\end{gathered}
$$


Then

$$
\begin{aligned}
V^{\prime}(t) & =2 \alpha_{f} \alpha_{p} \gamma_{1}\left(-\int_{0}^{\ell_{1}} \int_{0}^{\ell_{2}}\left(\partial_{x} e^{f}(t, x, y)\right)^{2} d y d x\right. \\
& \left.+\gamma \int_{0}^{\ell_{2}} e^{f}\left(t, \ell_{1}, y\right)\left(e^{p}\left(t, \ell_{1}, y\right)-e^{f}\left(t, \ell_{1}, y\right)\right) d y\right) \\
& -\beta_{f} \alpha_{p} \gamma_{1} \int_{0}^{\ell_{1}}\left(e^{f}\right)^{2}\left(t, x, \ell_{2}\right) d x \\
& +2 \alpha_{p} \gamma_{1} \int_{0}^{\ell_{1}} \int_{0}^{\ell_{2}} e_{f}(t, x, y) L_{f} e^{f}\left(t, x, \ell_{2}\right) d y d x \\
& +2 \alpha_{p} \alpha_{f} \gamma\left(-\int_{0}^{\ell_{1}} \int_{0}^{\ell_{2}}\left(\partial_{x} e^{p}(t, x, y)\right)^{2} d y d x\right. \\
& \left.+\gamma_{1} \int_{0}^{\ell_{2}} e^{p}\left(t, \ell_{1}, y\right)\left(e^{f}\left(t, \ell_{1}, y\right)-e^{p}\left(t, \ell_{1}, y\right)\right) d y\right) \\
& -\beta_{p} \alpha_{f} \gamma \int_{0}^{\ell_{1}}\left(e^{p}\right)^{2}(t, x, 0) d x \\
& +2 \alpha_{f} \gamma \int_{0}^{\ell_{1}} \int_{0}^{\ell_{2}} e^{p}(t, x, y) L_{p} e^{p}(t, x, 0) d y d x .
\end{aligned}
$$

By using the Poincaré inequality given in [14, Theorem 13.6.9 pages 426] then there exists $c=\left(\left(\ell_{1}+\ell_{2}\right)^{2} / \pi^{2}\right)>0$ such that

$$
\begin{aligned}
& \int_{0}^{\ell_{1}} \int_{0}^{\ell_{2}}\left(e^{p}(t, x, y)\right)^{2} d y d x \\
& \quad \leqslant c \int_{0}^{\ell_{1}} \int_{0}^{\ell_{2}}\left(\partial_{x} e^{p}(t, x, y)\right)^{2} d y d x .
\end{aligned}
$$

Moreover, we have the same inequality for $e^{f}$. Then

$$
\begin{aligned}
V^{\prime}(t) & \leqslant-2 \alpha_{f} \alpha_{p} \gamma_{1} \gamma \int_{0}^{\ell_{2}}\left(e^{f}\left(t, \ell_{1}, y\right)-e^{p}\left(t, \ell_{1}, y\right)\right)^{2} d y \\
& -2 \frac{\alpha_{f} \alpha_{p} \gamma_{1}}{c} \int_{0}^{\ell_{1}} \int_{0}^{\ell_{2}}\left(e^{f}(t, x, y)\right)^{2} d y d x \\
& -\beta_{f} \alpha_{p} \gamma_{1} \int_{0}^{\ell_{1}}\left(e^{f}\right)^{2}\left(t, x, \ell_{2}\right) d x \\
& +2 \int_{0}^{\ell_{1}} \int_{0}^{\ell_{2}} e_{f}(t, x, y) L_{f} e^{f}\left(t, x, \ell_{2}\right) d y d x \\
& -2 \frac{\alpha_{f} \alpha_{p} \gamma}{c} \int_{0}^{\ell_{1}} \int_{0}^{\ell_{2}}\left(e^{p}(t, x, y)\right)^{2} d y d x \\
& -\beta_{p} \alpha_{f} \gamma \int_{0}^{\ell_{1}}\left(e^{p}\right)^{2}(t, x, 0) d x \\
& +2 \int_{0}^{\ell_{1}} \int_{0}^{\ell_{2}} e^{p}(t, x, y) L_{p} e^{p}(t, x, 0) d y d x .
\end{aligned}
$$

Using young's inequality, we have for all $\epsilon_{2}, \epsilon_{3}>0$

$$
\begin{aligned}
& 2 \int_{0}^{\ell_{1}} \int_{0}^{\ell_{2}} e^{f}(t, x, y) L_{f} e^{f}\left(t, x, \ell_{2}\right) d y d x \\
& \leqslant \epsilon_{2} \int_{0}^{\ell_{1}} \int_{0}^{\ell_{2}}\left(e^{f}(t, x, y) L_{f}\right)^{2} d y d x \\
& +\frac{\ell_{2}}{\epsilon_{2}} \int_{0}^{\ell_{1}}\left(e^{f}\right)^{2}\left(t, x, \ell_{2}\right) d x,
\end{aligned}
$$

$$
\begin{aligned}
& 2 \int_{0}^{\ell_{1}} \int_{0}^{\ell_{2}} e^{p}(t, x, y) L_{p} e^{p}(t, x, 0) d y d x \\
& \leqslant \epsilon_{3} \int_{0}^{\ell_{1}} \int_{0}^{\ell_{2}}\left(e^{p}(t, x, y) L_{p}\right)^{2} d y d x+ \\
& \frac{\ell_{2}}{\epsilon_{3}} \int_{0}^{\ell_{1}}\left(e^{p}\right)^{2}(t, x, 0) d x,
\end{aligned}
$$

Taking $\epsilon_{2}=\frac{\ell_{2}}{\beta_{f} \alpha_{p} \gamma_{1}}$ and $\epsilon_{3}=\frac{\ell_{2}}{\beta_{p} \alpha_{f} \gamma}$

$$
\begin{aligned}
V^{\prime}(t) & \leqslant-2 \frac{\alpha_{f} \alpha_{p} \gamma_{1}}{c} \int_{0}^{\ell_{1}} \int_{0}^{\ell_{2}}\left(e^{f}(t, x, y)\right)^{2} d y d x \\
& +\frac{\ell_{2}}{\beta_{f} \alpha_{p} \gamma_{1}} \int_{0}^{\ell_{1}} \int_{0}^{\ell_{2}}\left(e_{f}(t, x, y) L_{f}\right)^{2} d y d x \\
& -2 \frac{\alpha_{f} \alpha_{p} \gamma}{c} \int_{0}^{\ell_{1}} \int_{0}^{\ell_{2}}\left(e^{p}(t, x, y)\right)^{2} d y d x \\
& +\frac{\ell_{2}}{\beta_{p} \alpha_{f} \gamma} \int_{0}^{\ell_{1}} \int_{0}^{\ell_{2}}\left(e^{p}(t, x, y) L_{p}\right)^{2} d y d x
\end{aligned}
$$

The observers gain satisfy condition (6) then there exists $\rho=\rho\left(\alpha_{f} \beta_{f}, \alpha_{p} \beta_{p}, c\right)>0$ such that

$$
V^{\prime}(t) \leqslant-\rho V(t)
$$

This ends the proof of this theorem.

\section{NUMERICAL ILLUSTRATIONS}

In this section, simulation results are presented to illustrate the convergence of the proposed observer for the DCMD model. We use real process parameters values given in the paper [6] which is provided by the Water Desalination and Reuse Center at KAUST. We consider $\Omega=\left(0, \ell_{1}\right) \times\left(0, \ell_{2}\right)$ where $\ell_{1}=0.001 \mathrm{~m}$ and $\ell_{2}=1 \mathrm{~m}$. The finite difference method is used for the numerical approximation. The Dirichlet boundary conditions are equal to $T_{f}=60 \mathrm{~K}$ and $T_{p}=$ $20 K$, respectively. The initial data are assumed to be equal to $f_{0}=10 \mathrm{~K}$ and $p_{0}=80 \mathrm{~K}$, where the initial conditions of the observer are given by $\hat{f}_{0}=13 \mathrm{~K}$ and $\hat{p}_{0}=7 \mathrm{~K}$. We assume that $N_{x}=100$ and $N_{y}=200$. The time step is equal to $\delta t=10^{-2}$. We fix the observer's gains to

$$
L_{f}=\frac{\alpha_{f} \alpha_{p}^{2} \gamma_{1}^{2} \beta_{f}}{4}, \quad L_{p}=\frac{\alpha_{p} \alpha_{f}^{2} \gamma^{2} \beta_{p}}{4},
$$

satisfying the exponential convergence condition given by (6). Figures 2 and 3 show the profile of the permeate and feed temperature for the state and estimate temperature. Moreover, figures 4 and 5 present the evolution for the feed and permeate temperature at $x=\ell_{1} / 2$ respectively for the final time. Figures 6 and 7 illustrate the $L^{2}-$ norm of error of estimation which verifies the effectiveness of the proposed observer.

\section{CONCLUSion}

This paper proposed an observer design for a system of advection diffusion equations coupled at the boundary and used to describe heat transfer in DCMD process. The observer estimates the feed and permeate temperatures distribution at the membrane. Sufficient conditions for the convergence 

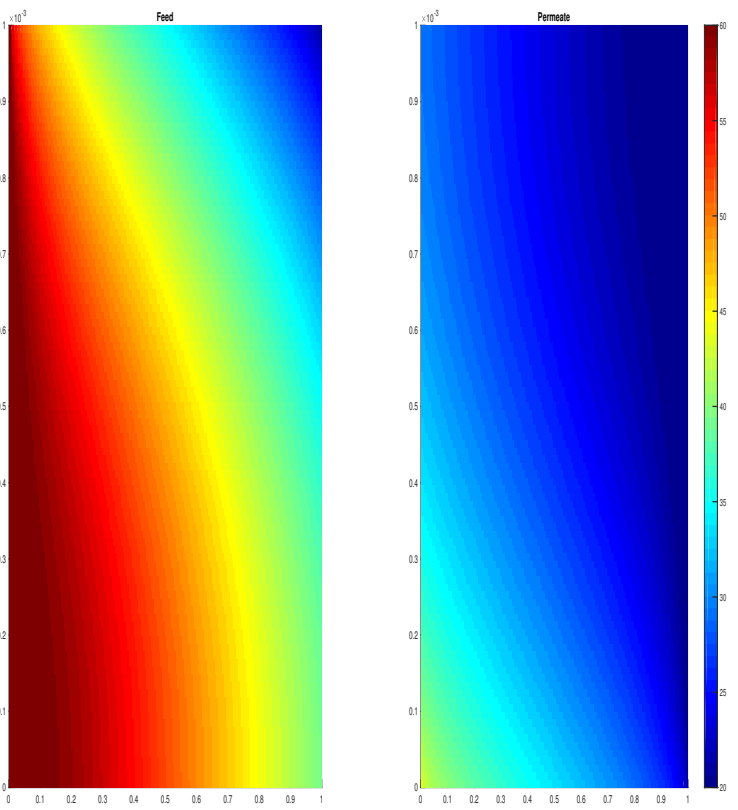

Fig. 2. The profile of temperature for the feed and the permeate temperature at final time.
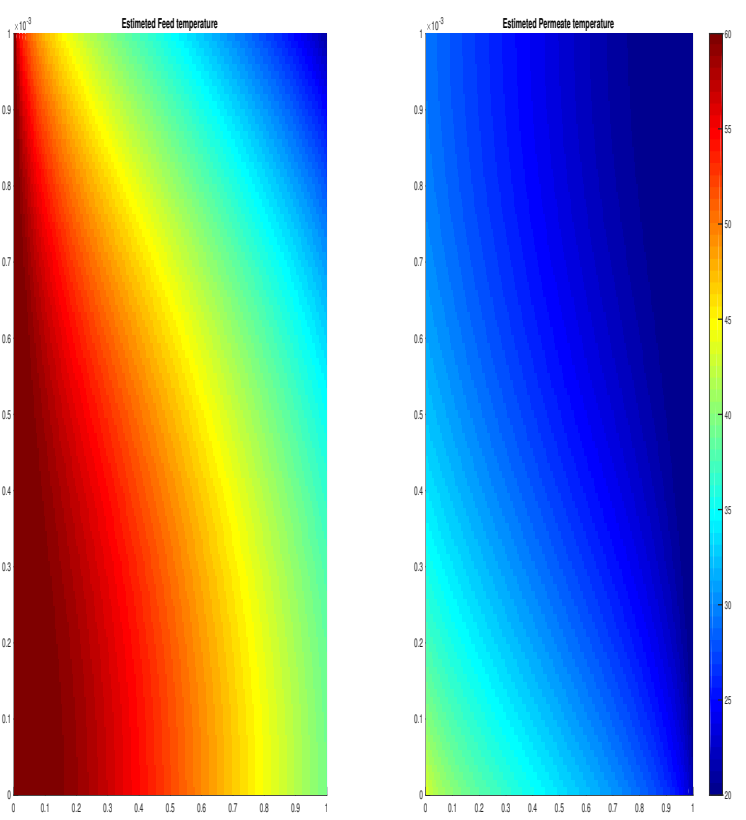

Fig. 3. The profile of the estimated temperature for the feed and the permeate temperature at final time.

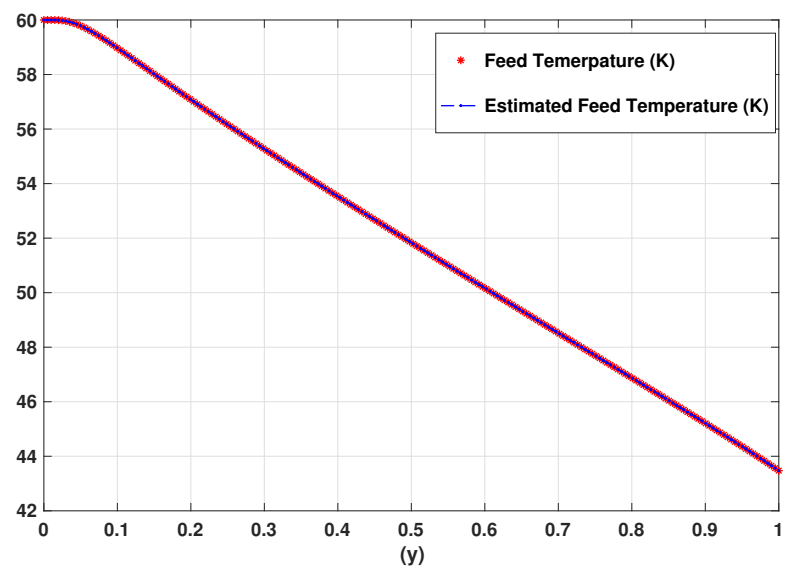

Fig. 4. The evolution of the feed temperature at $x=\ell_{1} / 2$ at final time.

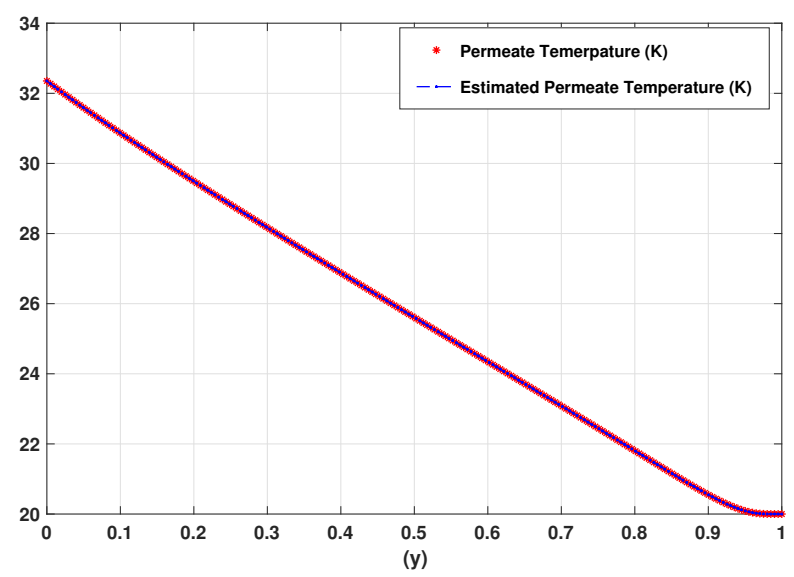

Fig. 5. The evolution of the permeate temperature at $x=\ell_{1} / 2$ at final time.

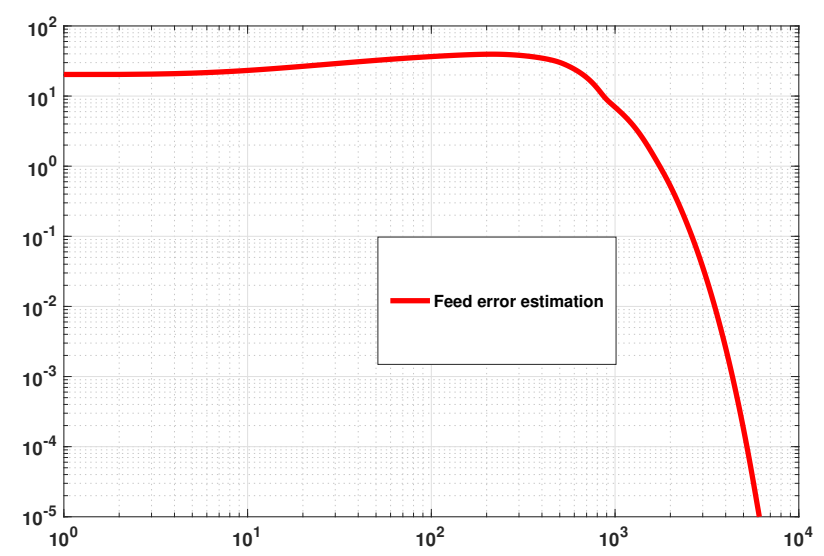

Fig. 6. The $L^{2}-$ norm of the error of estimation for the feed temperature. 


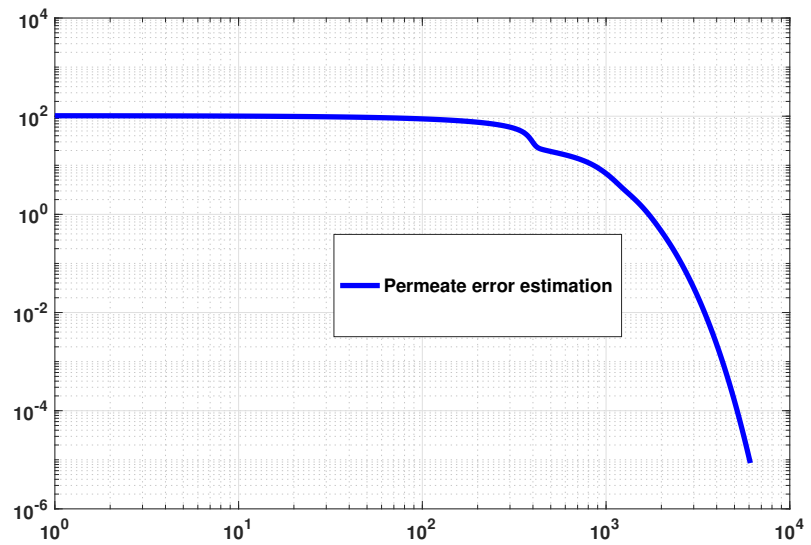

Fig. 7. The $L^{2}-$ norm of the error of estimation for the permeate temperature.

of the observer are proposed using the Lyapunov theory. Simulation results based on real physical parameters values are presented to illustrate the convergence of the proposed observer.

\section{REFERENCES}

[1] L. M. Camacho, L. Dumée, J. Zhang, J.-d. Li, M. Duke, J. Gomez, and S. Gray, "Advances in membrane distillation for water desalination and purification applications," Water, vol. 5, no. 1, pp. 94-196, 2013

[2] L. Francis, N. Ghaffour, A. Alsaadi, S. Nunes, and G. Amy, "Performance evaluation of the dcmd desalination process under bench scale and large scale module operating conditions," Journal of Membrane Science, vol. 455, pp. 103-112, 2014.

[3] F. Eleiwi and T. M. Laleg-Kirati, "Membrane distillation process modeling: Dynamical approach," International Journal of Chemical, Nuclear, Metallurgical and Materials Engineering, vol. 8, no. 6, pp. 479 - 484, 2014.

[4] M. Gryta, Water Desalination by Membrane Distillation, Desalination, Trends and Technologies. InTech, (2011).

[5] A. M. Karam, A. S. Alsaadi, N. Ghaffour, and T. Laleg-Kirati, "Analysis of direct contact membrane distillation based on a lumpedparameter dynamic predictive model," Desalination, vol. 402, pp. 50 61, 2017

[6] F. Eleiwi, N. Ghaffour, A. S. Alsaadi, L. Francis, and T. M. LalegKirati, "Dynamic modeling and experimental validation for direct contact membrane distillation (DCMD) process," Desalination, vol. 384, pp. 1-11, 2016.

[7] A. M. Karam, Reduced-Order Dynamic Modeling, Fouling Detection, and Optimal Control of Solar-Powered Direct Contact Membrane Distillation. PhD thesis, 2016.

[8] F. Eleiwi, Dynamic Modeling and Control of Distributed Heat Transfer Mechanisms: Application to a Membrane Distillation Module. PhD thesis, 2015.

[9] C. McFall, A. Bartman, P. Christofides, and Y. Cohen, "Control of a reverse osmosis desalination process at high recovery," pp. 2241-2247, June 2008.

[10] A. Gambier, A. Wellenreuther, and E. Badreddin, "Optimal control of a reverse osmosis desalination plant using multi-objective optimization,' pp. 1368-1373, Oct 2006.

[11] A. Bartman, C. McFall, P. Christofides, and Y. Cohen, "Model predictive control of feed flow reversal in a reverse osmosis desalination process," American Control Conference, pp. 4860-4867, June 2009.

[12] L. Jadachowski, T. Meurer, and A. Kugi, "Backstepping observers for linear pdes on higher-dimensional spatial domains," Automatica, vol. 51, pp. 85-97, 2015 .

[13] M. Khayet, Advanced Membrane Technology and Applications. John Wiley \& Sons, Inc., (2008).

[14] M. Tucsnak and G. Weiss, Observation and control for operator semigroups. Birkhauser, Birkhauser Verlag, Basel, 2009. 